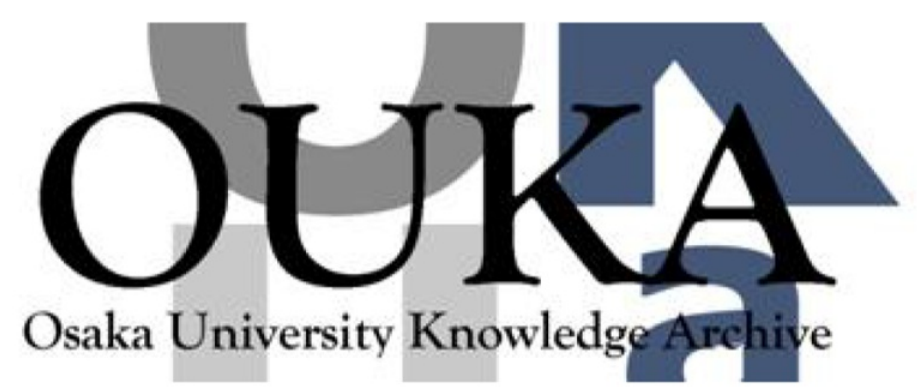

\begin{tabular}{|c|l|}
\hline Title & $\begin{array}{l}\text { Optical properties of di-substituted acetylene } \\
\text { polymers }\end{array}$ \\
\hline Author(s) & $\begin{array}{l}\text { Tada, Kazuya; Hidayat, Rahmat; Hirohata, } \\
\text { Masaharu et al. }\end{array}$ \\
\hline Citation & $\begin{array}{l}\text { Proceedings of SPIE - The International Society } \\
\text { for Optical Eng ineering. 3145 p. 171-p. 178 }\end{array}$ \\
\hline Issue Date & $1997-12-01$ \\
\hline oaire:version & VoR \\
\hline URL & https://hdl. handle. net/11094/76946 \\
\hline rights & \\
\hline Note & \\
\hline
\end{tabular}

Osaka University Knowledge Archive : OUKA

https://ir. Library. osaka-u. ac. jp/

Osaka University 


\title{
Optical Properties of Di-substituted Acetylene Polymers
}

\author{
Kazuya Tada, Rahmat Hidayat, Masaharu Hirohata, Hirotake Kajii, Satoshi Tatsuhara, Akihiko Fujii, \\ Masanori Ozaki, Masahiro Teraguchi*, Toshio Masuda* and Katsumi Yoshino \\ Department of Electronic Engineering, Osaka University, 2-1 Yamada-Oka, Suita, Osaka 565, Japan \\ * Division of Polymer Chemistry, Kyoto University, Yoshida-Honmachi, Sakyoku, Kyoto 606, Japan
}

\begin{abstract}
Optical properties and electronic energy structures of di-substituted acetylene polymers have been studied. Strong blue and green photoluminescence and electroluminescence have been observed in the poly(diphenylacetylene) (PDPA) derivatives and poly(1-alkyl-2-phenylacetylene) (PAPA) derivatives, contrary to trans-polyacetylene, in which only very weak photoluminescence are observed at infrared region. The dependence of optical properties of the di-substituted polyacetylene derivatives such as photoluminescence intensity and electroluminescence efficiency on the molecular structure of substituents and the change of optical properties upon electrochemical doping and their interpretation by soliton model have also been discussed. Spectral narrowing in photoluminescence of some di-substituted polyacetylenes have also been studied.
\end{abstract}

\section{INTRODUCTION}

Recently, conducting polymers with extensive $\pi$-conjugation in their main chain have attracted much attentions as materials for electronic and optoelectronic devices such as electroluminescent devices ${ }^{1-3)}$ and photocells, ${ }^{47)}$ because of their high quantum electron/photon conversion efficiency and advantages for fabrication of thin films with large area. They have attracted much attentions from not only such practical viewpoints but also fundamental viewpoints because they exhibits numerous unique phenomena such as drastic changes in electrical, optical and magnetic properties upon doping.

Polyacetylene is a conducting polymer with most simple backbone structure and many studies were carried out on the characteristics of this polymer. ${ }^{8)}$ Although practical applications of polyacetylene and their derivatives such as an electrode of a secondary battery were attempted but they have not been successful. On the other hand, we proposed for the first time to apply a trans-polyacetylene derivative, perfluoroalkylated poly(diphenylacetylene) to electroluminescent devices, and also demonstrated strong green photoluminescence. ${ }^{\text {i) }}$

In the early stage of study of polyacetylene, Lauchlan et al. reported the absence of photoluminescence in trans-polyacetylene and interpreted the result based on the soliton model. ${ }^{10)}$ The lack of the proposals of electroluminescent device with transpolyacetylene derivatives before us may be partly due to a speculation associated with this interpretation that efficient luminescence will not be expected in any trans-polyacetylene derivatives . 
However, we ${ }^{11)}$ and Fitchen ${ }^{12)}$ have observed weak photoluminescence at infrared region in trans-polyacetylene, suggesting that not the soliton dynamics but the relative position of $1^{1} B_{u}$ and $2^{1} A_{g}$ metastable states in energy is important to interpret the luminescence of this polymer. That is, in trans-polyacetylene, the metastable $1^{1} \mathrm{~B}_{u}$ state is higher than $2^{1} \mathrm{~A}_{\mathrm{g}}$ state in energy, resulting in the weak photoluminescence. The subststituents may influence on the energy levels of $2^{l} \mathrm{~A}_{g}$ and $1^{1} \mathrm{~B}_{u}$ states.

In this article, we report optical properties and electronic energy structures of di-substituted acetylene polymers.

\section{EXPERIMENTAL}

Various di-substituted acetylene polymers such as poly(1-phenyl-2-p-n-butyl phenyl acetylene) (PDPA-nBu), poly(1-phenyl2-p-tert-butylphenyl acetylene) (PDPA-tBu), poly(1-phenyl-2-p-adamantylphenyl acetylene) (PDPA-Ad), poly(1-phenyl-2-ptrimethylsilylphenyl acetylene) (PDPA-SiMe ${ }_{3}$ ), poly(1-phenyl-2-p-tri(isopropyl)silyl phenylacetylene) (PDPA-SiiPr ${ }_{3}$ ), poly(1phenyl-2-p-triphenylsilylphenyl acetylene), ( $\mathrm{PDPA}-\mathrm{SiPh}_{3}$ ), poly(1-phenyl-2-m-trimethylsilylphenyl acetylene) (PDPA-mSiMe ${ }_{3}$ ), poly(1-phenyl-2-p-phenoxyphenyl acetylene) (PDPA-OPh), poly(1-methyl-2-phenyl acetylene) (PMePA), poly(1-ethyl-2-phenyl acetylene) (PEtPA), poly(1-n-hexyl-2-phenyl acetylene) (PHxPA) and poly(1-methyl-2-naphthyl acetylene) (PMeNA) were studied here. The molecular structures of these polymers are shown in Fig. 1. The synthesis of these di-substituted acetylene polymers are described elsewhere. ${ }^{13)}$ These polymers are soluble in common organic solvents such as chloroform.

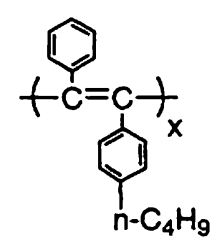

PDPA-nBu<smiles>[X]C(=Cc1ccccc1C(C)(C)C)c1ccc([Si](C)(C)C(C)C)cc1</smiles>

$\mathrm{PDPA}_{\mathrm{SiiPr}}$

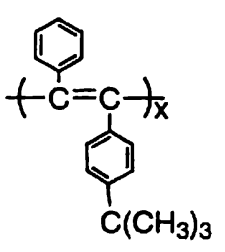

PDPA-tBu<smiles>[X]C(C)(C)c1ccc(C(C)(C)C)cc1</smiles>

PDPA-SiPh 3

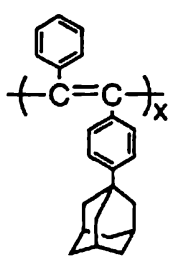

PDPA-Ad<smiles>[X]C(C)(C)C(=Cc1ccccc1C(C)(C)C)c1cccc([SiH2]C)c1</smiles>

PDPA-mSiMe ${ }_{3}$<smiles>[X]C(C)(C)Cc1cc(C)ccc1C=C(C)C(C)(C)C</smiles>

PDPA-SiMe 3<smiles>[X]c1ccc(Oc2ccccc2)cc1/C=C/c1ccccc1C(C)(C)C</smiles>

PDPA-OPh<smiles>[X]C(C)(C)C(C)=CC=C1C=CC=C1</smiles>

PMePA<smiles>[X]C(C)(CC)C(C)(C)C(C)(C)C</smiles>

PEtPA

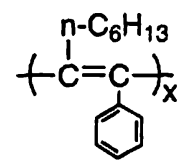

PHXPA

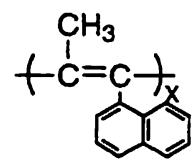

PMeNA

Fig. 1 Molecular structures of di-substituted acetylene polymers studied here. 
Thin films were prepared by spin coating or casting a chloroform solution containing the conducting polymer on appropriate substrates such as quartz plates or In-Sn oxide (ITO) coated quartz plates (transparent electrodes).

Optical absorption and photoluminescence spectra were measured using spectrophotometer (HP 8452 or Hitachi 330) and fluorescence spectrophotometer (Hitachi F2000), respectively.

Electrochemical measurements such as cyclic voltammetry were carried out using potentiostat (Hokuto-Denko HA501) and function generator (Hokuto-Denko HB 104) in a dry-box filled with Ar gas. Used three-electrode electrochemical cell was filled with acetonitrile containing tetrabutylammonium tetrafluoroborate $\left(\mathrm{TBABF}_{4}\right)$ as supporting salt, and had a casted film on ITOcoated glass, an Ag wire and a Pt plate as working electrode (WE), reference electrode (RE) and counter electrode (CE), respectively.

ESR study was carried out with Bruker ESP-300. Spin density was measured using DPPH as the reference.

The electroluminescent devices were fabricated by depositing a $\mathrm{Mg}$-In alloy onto a spin coated polymer films on the ITOcoated glass plates or ITO-coated quartz substrates. The emission area were approximately $6 \mathrm{~mm}^{2}$. Electroluminescent characteristics were studied either under vacuum as room temperature or in liquid $\mathrm{N}_{2}$ by a method described elsewhere. ${ }^{2.3 .9}$ )

The emission spectra at strong excitation were measured using a spectrophotometer (Hamamatsu Photonics PMA-11) upon irradiation of laser pulse with $10 \mathrm{~ns}$ in width and $355 \mathrm{~nm}$ in wavelength produced by THG of a Nd:YAG laser light.
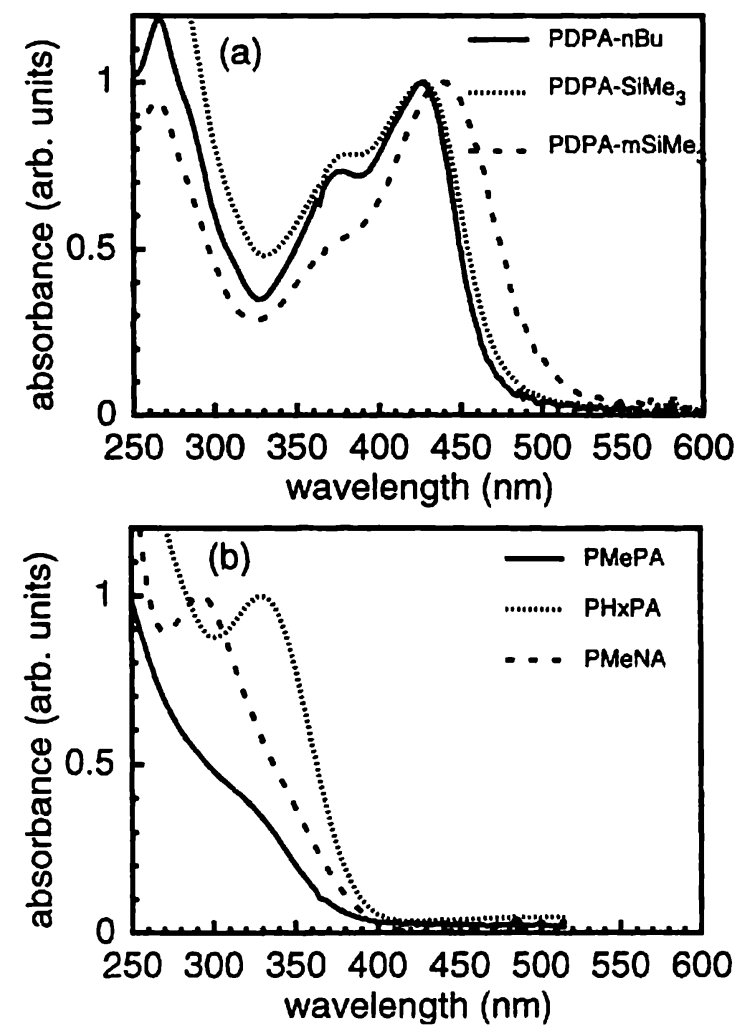

Fig. 2 Optical absorption spectra of poly(diphenylacetylene) derivatives (a) and poly(1-alkyl-2phenyl acetylene) derivatives. 


\section{RESULTS AND DISCUSSION}

Optical absorption spectra of poly(diphenylacetylene) (PDPA) derivatives and poly(1-alkyl-2-phenyl acetylene) (PAPA) derivatives are shown in Figs. 2(a) and (b), respectively. It is clearly indicated that PDPA derivatives such as PDPA-nBu, $\mathrm{PDPA}_{-} \mathrm{SiMe}_{3}$ and PDPA-mSiMe ${ }_{3}$ have the absorption edge at longer wavelength than PAPA derivatives such as PMePA, PHXPA and PMeNA.

The band-gap energies of these polymers can be evaluated from Fig. 2 using ( $\alpha h v)^{2}-h v$ plot where $\alpha, h$ are the absorbance at frequency $v$ and the Planck's constant, respectively, and are summarized in Table 1 . The energies of highest occupied molecular orbital (HOMO) corresponding to the valence band top evaluated from cyclic voltammetry using Ag wire as a reference electrode are also listed in this table.

The band-gap energies of various PDPA derivatives with side group at para-position are almost the same regardless of the molecular structure of the side group. However, it should be noted that the position of the side group influences a little to the band gap energy. That is, the band gap energy of PDPA-mSiMe ${ }_{3}$ having the same substituent with PDPA-SiMe ${ }_{3}$ at different position (meta-position) is $2.57 \mathrm{eV}$ and significantly smaller than that of PDPA-SiMe ${ }_{3}(2.70 \mathrm{eV})$. The band-gap energy of perfluoroalkyl poly(diphenylacetylene) (PFPDA) previously reported is $2.7 \mathrm{eV}$ and also coincide with those of PDPA derivatives studied here.

The molecular structures of the substituents in poly(1-alkyl-2-phenylacetylene) derivatives should influence the electronic energy structure. The energy of HOMO of poly(1-alkyl-2-phenyl acetylene)s becomes lower with increasing alkyl chain length, and that of PMePA is higher than that of PMeNA.

Table 1. HOMO level, band gap, PL and EL peaks and Stokes shift value of di-substituted acetylene polymers.

\begin{tabular}{llllll}
\hline Polymer & $\begin{array}{l}\text { Band gap } \\
(\mathrm{eV})\end{array}$ & $\begin{array}{l}\text { HOMO vs } \\
\text { vacuum level } \\
(\mathrm{eV})\end{array}$ & $\begin{array}{l}\text { PL peak } \\
(\mathrm{eV})\end{array}$ & $\begin{array}{l}\text { EL peak } \\
(\mathrm{eV})\end{array}$ & $\begin{array}{l}\text { Stokes shift } \\
(\mathrm{eV})\end{array}$ \\
\hline PDPA-nBu & 2.73 & -5.6 & 2.38 & 2.40 & 0.35 \\
PDPA-tBu & 2.71 & -5.7 & 2.36 & 2.43 & 0.35 \\
PDPA-Ad & 2.67 & -5.6 & 2.31 & 2.25 & 0.36 \\
PDPA-SiMe $_{3}$ & 2.70 & -5.75 & 2.36 & 2.40 & 0.34 \\
PDPA-SiiPr $_{3}$ & 2.74 & -5.9 & 2.38 & 2.35 & 0.36 \\
PDPA-SiPh $_{3}$ & 2.71 & -5.9 & 2.34 & 2.38 & 0.37 \\
PDPA-mSiMe 3 & 2.57 & -5.75 & 2.29 & 2.23 & 0.28 \\
PDPA-OPh & 2.66 & -5.7 & 2.30 & 2.25 & 0.36 \\
PMePA & 3.42 & -5.9 & 2.63 & 2.58 & 0.79 \\
PEtPA & 3.35 & -6.0 & 2.66 & 2.70 & 0.69 \\
PHXPA & 3.35 & -6.1 & 2.70 & 2.76 & 0.65 \\
PMeNA & 3.40 & -6.1 & 2.61 & 2.48 & 0.79 \\
\hline
\end{tabular}




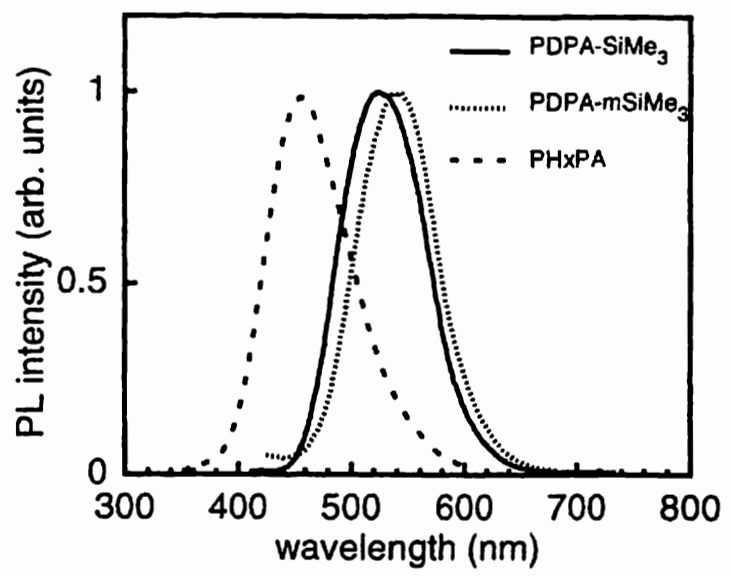

Fig. 3 Photoluminescence spectra of PDPA-SiMe ${ }_{3}, \mathrm{PDPA}-\mathrm{mSiMe}_{3}$ and PHxPA.

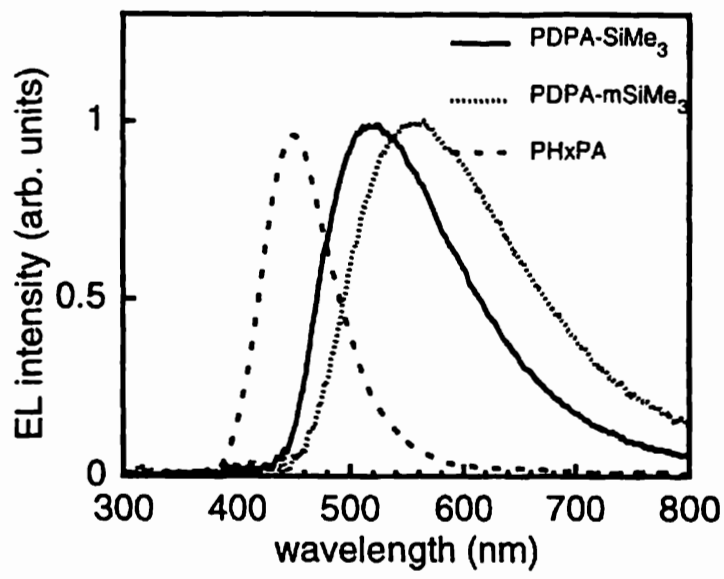

Fig. 4 Electroluminescence spectra of PDPA-SiMe ${ }_{3}, \mathrm{PDPA}_{\mathrm{mSiMe}}$ and PHxPA.

Figure 3 shows the photoluminescence spectra of the di-substituted acetylene polymers such as PDPA-SiMe $e_{3}, \mathrm{PDPA}_{-\mathrm{mSiMe}}$ and PHxPA. PDPA derivatives with various substituents show strong green photoluminescence with the peak at almost the same photon energy. Strong blue or blue-green photoluminescences are observed in PAPA derivatives and the peak energy of them are blue-shifted with increasing alkyl chain length. Stokes shift, which is the difference between the band gap energy and the photoluminescence peak energy, of the di-substituted polyacetylenes are summarized also in Table 1 as well as photoluminescence peak energies. All of these polymers show relatively large Stokes shift as observed in trans-polyacetylene, reflecting their backbone structure.

Electroluminescence spectra of the device with the di-substituted acetylene polymers are shown in Fig. 4. The electroluminescence spectra of the polymers almost coincide with their photoluminescence spectra. The electroluminescence peak energy of the polymers are summarized also in Table 1, and almost coincide with photoluminescence peak energy.

Unique dependence of the intensities on the molecular structure of the side groups were found in both the electroluminescence and photoluminescence of the di-substituted polyacetylenes. Figure 5 shows the photoluminescence intensity and the relative electroluminescence efficiency of the PDPA derivatives with substituent containing Si atom. Among para-substituted PDPA 


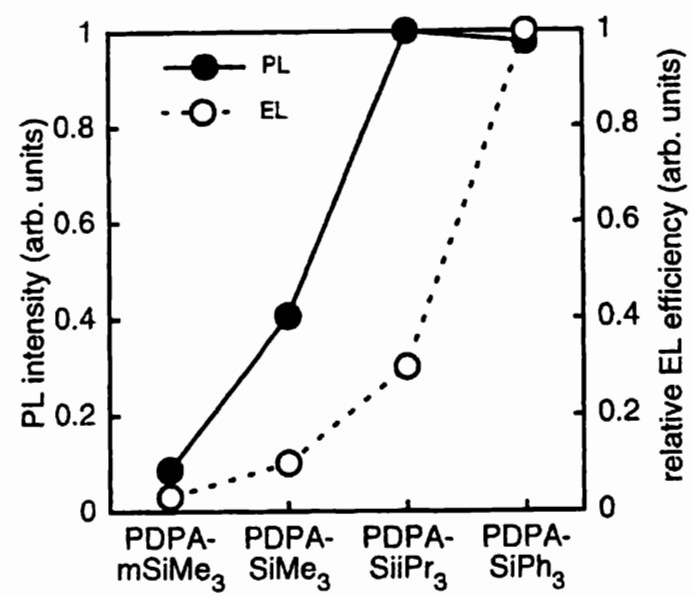

Fig. 5 Photoluminescence intensity and relative electroluminescence efficiency of the poly(diphenylacetylene) derivatives with substituent containing Si atom.

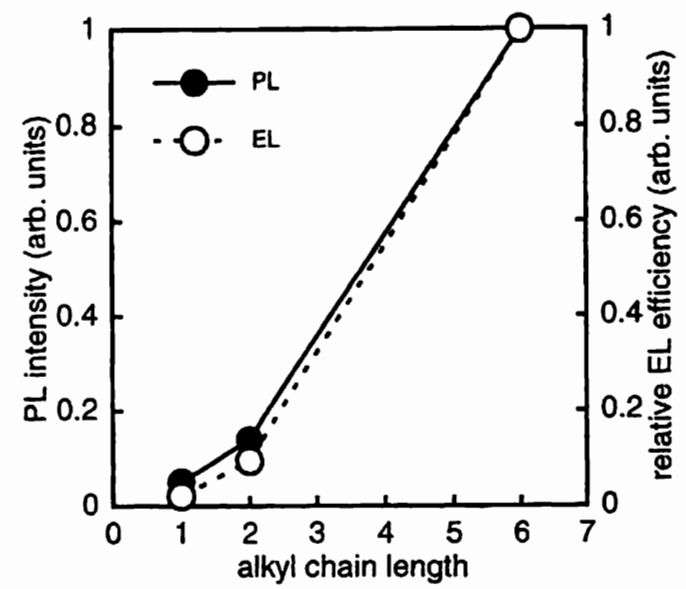

Fig. 6. Dependence of Photoluminescence intensity and relative electroluminescence efficiency of poly(1alkyl-2-phenyl acetylene)s on alkyl chain length.

derivatives, $\mathrm{PDPA}-\mathrm{SiPh}_{3}$ and PDPA-SiiPr 3 show stronger photoluminescence and electroluminescence than $\mathrm{PDPA}-\mathrm{SiMe}_{3}$ with smaller substituents. This can be tentatively understood in terms of steric hindrance between substituents. That is, in PDPA derivatives with larger substituents, more efficient confinement of the exciton or exciton-polaron in the main chain should take place due to larger streric hindrance between substituents, resulting in stronger luminescence. The photoluminescence intensity and electroluminescence intensity of PDPA-mSiMe ${ }_{3}$ are significantly smaller than those of PDPA-SiMe, suggesting that the position of the substituent also affect to the exciton confinement nature.

The photoluminescence intensity and electroluminescence efficiency of poly(1-alkyl -2-phenyl acetylene)s increase with increasing alkyl chain length, as shown in Fig. 6 . This can also be tentatively interpreted in terms of exciton confinement effect due to steric hindrance between the alkyl side chain and the polymer main chain.

It is important to check whether the solitons exist or not in these fluorescent di-substituted polyacetylenes. Upon electrochemical $\mathrm{BF}_{4}$ - doping (p-type doping) to some di-substituted polyacetylenes such as PDPA-nBu, we have observed evolution of optical absorption at the photon energy near the half of the band-gap energy as well as the suppression of the inter-band absorption, 


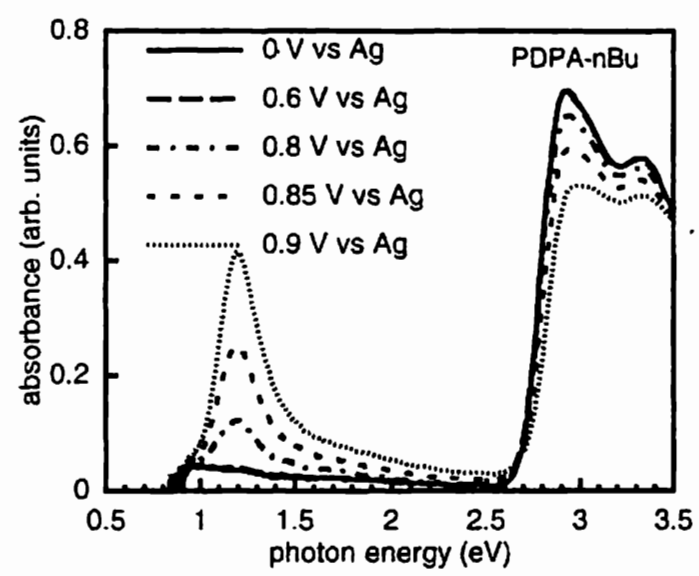

Fig. 7. Change of optical absorption spectra of PDPA-nBu upon electrochemical $\mathrm{BF}_{4}$ - doping.

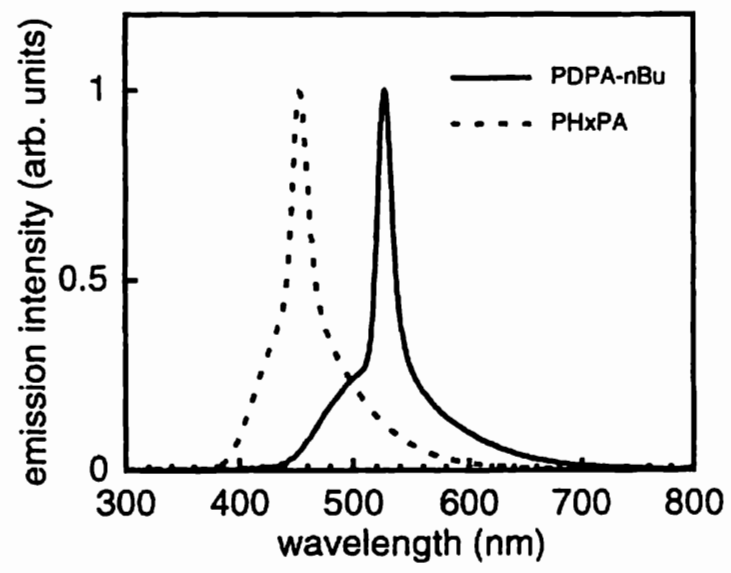

Fig. 8. Emission spectra of PDPA-nBu and PHxPA at strong excitation.

which is similar phenomenon observed in trans-polyacetylene, ${ }^{14)}$ as shown in Fig. 7. Moreover, until certain doping level, while significant evolution of the absorption at the half-band-gap energy takes place, significant increase of spin density was not observed, indicating the creation of spinless charged species or solitons in the di-substituted acetylene polymers.

Upon highly intense excitation, spectral narrowing of the photoluminescence ${ }^{15-17)}$ was observed in some di-substituted polyacetylenes due to sumulated emission. The emission spectra of PDPA-nBu and PHxPA at strong excitation are shown in Fig. 8.

\section{SUMMARY}

The experimental results are summarized as follows.

(1) Strong blue and green photoluminescence and electroluminescence were observed in various di-substituted acetylene polymers. Poly(1-alkyl-2-phenyl acetylene) derivatives show blue or blue-green luminescence depending on substituents and poly(diphenylacetylene) derivatives show green luminescence.

(2) The photoluminescence intensity and electroluminescence efficiency in the di-substituted acetylene polymers are strongly 
dependent on the molecular structure of the substituents. This was explained in terms of change in exciton confinement due to steric hindrance effect.

(3) Creation of solitons in the di-substituted acetylene polymers were confirmed by checking the change of optical absorption spectrum and spin density upon electrochemical doping.

(4) Spectral narrowing was observed in photoluminescence of some di-substituted acetylene polymers such as PDPA-nBu and PHxPA by strong excitation.

\section{ACKNOWLEDGMENT}

This work was financially supported by the Research for the Future Program of the Japan Society of the Promotion of Science (Project No. JSPS-RFTF96P00206)

\section{REFERENCES}

1. J. H. Burroughes, D. D. C. Bradley, A. R. Brown, R. N. Marks, K. Mackay, R. H. Friend, P. L. Burns and A. B. Holmes: Nature 347 (1990) 539.

2. Y. Ohmori, M. Uchida, K. Muro and K. Yoshino: Jpn. J. Appl. Phys. 30 (1991) L1938.

3. Y. Ohmori, M. Uchida, K. Muro and K. Yoshino: Jpn. J. Appl. Phys. 30 (1991) L1941.

4. G. Yu, K. Pakbaz and A. J. Heeger: Appl. Phys. Lett. 64 (1994) 3422.

5. A. A. Zakhidov and K. Yoshino: J. Soc. Electrical Mater. Eng. 4 (1995) 43.

6. J. J. M. Halls, C. A. Walsh, N. C. Greenham, E. A. Marseglla, R. H. Friend, S. C. Moratti and A. B. Holmes: Nature 376 (1995) 498.

7. K. Tada, M. Onoda, A. A. Zakhidov and K. Yoshino: Jpn. J. Appl. Phys. 36 (1997) L306.

8. A. J. Heeger, S. Kivelson, J .R. Schriefer and W. P. Su: Rev. Mod. Phys. 60 (1988) 781.

9. K. Tada, H. Sawada, J. Kyokane and K. Yoshino: Jpn. J. Appl. Phys. 34 (1995) L1083.

10. L. Lauchlan, S. Etemad, T. C. Chung, A. J. Heeger and A. G. MacDiarmid: Phys. Rev. B 24 (1981) 3701.

11. K. Yoshino, S. Hayashi, Y. Inuishi, K. Hattori and Y. Watanabe: Solid State Commun. 46 (1983) 583.

12. D. B. Fitchen: Synth. Metals 9 (1984) 341.

13. H. Shirakawa, T. Masuda and T. Takeda: The Chemistry of Triple-Bonded Functional Groups, ed. S. Patai (John Wiley \& Sons, 1994) Chap.17, p. 945.

14. T. C. Chung, F. Moraes, J. D. Flood and A. J. Heeger: Phys. Rev. B 29 (1984) 2341.

15. N. Tessler, G. J. Denton and R. H. Friend: Nature 382 (1996) 695.

16. F. Hide, M. A. Diaz-Garcia, B. J. Schwartz, M. R. Anderson, Q. Pei and A. J. Heeger: Science 273 (1996) 1833.

17. S. V. Frolov, W. Gellermann, M. Ozaki, K. Yoshino and Z. V. Vardeny: Phys. Rev. Lett. 78 (1997) 729. 